\title{
Habiter la mobilité : le train comme terrain de réflexion
}

\author{
Jean-Baptiste Frétigny \\ Doctorant contractuel à l'université Paris I - UMR Géographie-cités et moniteur à I'ENS-UIm
}

«Version pre-print »

Résumé : Pour saisir la recomposition des territoires sous l'effet de la mobilité, cet article propose d'étudier l'habiter à micro-échelle des espaces de transport. A travers l'exemple de l'espace du train, il s'agit d'interroger la territorialité labile de ces espaces du déplacement en confrontant diverses conceptions du lieu à un travail d'enquête mené sur le cas italien. L'analyse des matériaux rassemblés montre que mobilité et habiter s'articulent dans une lutte des places et une cohabitation des passagers aux modalités originales, faisant de ces interstices mobiles des lieux de réinvention de l'urbanité.

Mots-clés : Habiter, mobilité, territorialité, lieu, transport, micro-échelle, Italie.

\section{English: Mobility and dwelling: train as ground of thought}

Abstract: To understand the reshaping of territories under the influence of mobility, this article speculates that microscale study of dwelling in spaces of mass-transit is operative. With the example of the space of the train, it questions the plastic territoriality of these spaces on the move through the discussion of several conceptions of territory and of a survey undertaken about the Italian case. A kind of war of places and a very special cohabitation are at stake in this combination of mobility and dwelling, making these mobile interstices places of reinvention of urbanity.

Key words: Dwelling, territory, place, network, transport, microscale, Italy.

\section{Italiano: Mobilità e abitare: il treno come terreno di riflessione.}

Riassunto: Per comprendere la ricomposizione dei territori per effetto della mobilità, quest'articolo propone un'analisi su micro scala dell'abitare degli spazi di trasporto. Si tratterà di analizzare, prendendo come esempio lo spazio del treno, l'effimera territorialità di questi spazi di spostamento, confrontando diverse concezioni del luogo a un lavoro d'indagine condotto sul caso italiano. L'analisi dei dati raccolti dimostra che mobilità e abitare si articolano in una lotta per il posto e in una coabitazione dei passegeri dalle modalità originali, che fa di questi interstizi mobili dei luoghi di reinvezione dell'urbanità.

Parole chiave: Territorio, luogo, rete, trasporto, micro-scala, Italia.

Les modèles territoriaux inspirés de l'Etat-nation ont longtemps occulté des territorialités plus complexes et moins délimitées. L'importance croissante du phénomène de mobilité oblige aujourd'hui à réinterroger ces figures archétypales du territoire et les représentations qui les sous-tendent, participant d'un champ d'interrogation théorique plus vaste que certains chercheurs n'ont pas hésité à qualifier de mobility turn en sciences sociales (Sheller et Urry, 2006). Cet article fait l'hypothèse que les espaces de transport ont une valeur heuristique particulière, non dans l'opposition mais dans l'articulation aux territoires les plus établis. Pour sortir d'une approche par la négative des espaces de transport, c'est une entrée par l'habiter qui est ici proposée.

L'amplification des mobilités a fréquemment été analysée comme un facteur de crise de l'habiter. Certaines approches récentes envisagent en revanche l'habiter selon une 
perspective moins sédentaire, comme un processus qui s'opère dans la pratique des lieux. Multipliant les espaces pratiqués, la mobilité favorise une diversification de l'habitat sous forme polytopique, jouant un rôle essentiel dans l'identité et le rapport à l'altérité des personnes mobiles (Stock, 2005).

La mobilité ne sera pas envisagée seulement comme un procédé pour passer à une activité, une translation d'un point $A$ à un point $B$, mais, de manière plus englobante, comme une activité en soi, qui s'accompagne d'un changement d'état de ceux qui la pratiquent. II faut donc envisager des espaces secondaires d'habitat dont la pratique est favorisée par excellence par la mobilité : les espaces de transport eux-mêmes.

C'est sur l'espace du train à micro-échelle que portera l'analyse, terrain d'investigation récemment défriché. Diverses études se sont penchées sur les pratiques à bord des passagers : usage des télécommunications, gestion du temps de voyage, construction d'ambiances particulières, voire des réseaux de sociabilité (Berry et al., 2010 ; Lyons et al., 2007 ; Watts, 2008 ; Bissell, 2009 et 2010 ; Lannéelle, 2004). Ces travaux ouvrent de nombreuses pistes mais le statut de cet espace du déplacement, contenant autant que contenu, n'y est pas interrogé. Quel sens donner à cet objet géographique, et en quoi peut-il contribuer à saisir les mutations territoriales suscitées par l'intensification des mobilités ?

Afin d'interroger diverses conceptions de l'espace du train, l'étude a mobilisé une palette assez large de matériaux concernant le cas italien. Elle articule une série d'entretiens, le résultat d'observations menées à bord des trains et un corpus de documents italiens à large diffusion, publicitaires, artistiques et juridiques. L'enquête proprement dite repose sur 17 entretiens semi-directifs approfondis, réalisés de mars à juin 2006 avec 22 passagers sélectionnés de manière aléatoire, 9 navetteurs et 13 voyageurs plus ou moins occasionnels. Réalisés in situ, les entretiens se sont déroulés en seconde classe, à bord de trains sillonnant pour l'essentiel le nord-est et le centre de la péninsule, en particulier entre Pise, Florence, Padoue et Venise. Chacun de ces entretiens a duré entre 30 et 45 minutes à bord des trains régionaux et plus de deux heures dans les trains longue distance. Le travail d'observation a été mené de janvier à juillet 2006 dans 80 trains de diverses catégories, sur des trajets d'envergure régionale à internationale (1).

Ce sont tout d'abord les conceptions du train comme non-lieu qui retiendront l'attention. Elles trahissent l'imprégnation urbaine de cet espace relevant à bien des égards d'une territorialité originale. II s'agira de voir ensuite comment cette territorialité permet d'habiter un tel espace mobile. L'étude montre que l'ancrage se construit autour de la place de chacun, mais de manière négociée et toujours provisoire. Les jeux de pouvoir qui s'opèrent dans le train révèlent enfin une urbanité qui interpelle à bien plus large échelle la construction des territoires.

\section{1 - L'espace de transport est-il un non-lieu ?}

Dans les entretiens, divers passagers érigent leur expérience du train comme une parenthèse dans leur existence. "C'est 30 heures de perdues chaque mois 》 déplore Cecilia, jeune consultante commerciale navetteuse, et Cosimo, militaire à la retraite, regrette d'y " être toujours en attente d'agir ». Ce thème du temps mort renvoie à une vision étanche de l'espace-temps du transport, sorte de point aveugle du monde social, dépourvu de toute signification. Doit-on en conclure qu'un tel espace constitue une sorte de négatif du lieu ? 


\section{L'espace du déplacement : parenthèse de la vie sociale?}

Dans un essai reposant sur sa propre expérience personnelle de voyageur, Marc Augé (1992) théorise une telle conception en qualifiant certaines installations spatiales, dont le train, de «non-lieux ». Leur fonction de mise en circulation accélérée des biens et des personnes les mettrait au ban du lieu anthropologique, "identitaire, relationnel et historique ». Dans le non-lieu, les interactions seraient réduites au minimum, à une sorte de " mode d'emploi » visant à assurer la bonne mise en place des conditions de circulation.

Ces apparents «modes d'emploi » sont présents dans tout l'espace du train sous forme d'instructions diverses. Mais ils renvoient à l'apprentissage d'un univers technique qui n'est pas neutre mais au contraire partie prenante du monde social, à l'image de la formule qui figure sur le chambranle des fenêtres des trains italiens : "è pericoloso sporgersi » («il est dangereux de se pencher au-dehors»). L'inscription, qui n'est pas inconnue des voyageurs de la SCNF, participe de la diffusion sociale et culturelle de normes de sécurité et de confort en Europe et dans le monde, à travers de multiples règlements et modèles de design. Les constructeurs ferroviaires, de plus en plus concentrés, la Commission européenne et l'Union internationale des chemins de fer donnent ainsi un sens à l'environnement socio-technique du train. Le libellé est d'ailleurs considéré avec une certaine distance par les voyageurs, comme un marqueur familier, repris par exemple par le chanteur Giuseppe Mango comme titre d'un de ses albums en 1982.

Cette négation de la socialité de l'espace du train fait étrangement écho à certains discours des premiers temps du chemin de fer en Europe. Aux yeux de certaines élites, le transport ferroviaire détruit l'espace intermédiaire qui sépare départ et arrivée et aboutit à une perte de « l'ici et maintenant » des lieux. Toute une littérature détaille les « pathologies » que susciteraient le voyage en train (Schivelbusch, 1990). Les passagers de première et de seconde classes nourrissent une nostalgie de l'authentique voyage que l'arrivée du chemin de fer et du passager de troisième classe auraient fait disparaître (Urbain, 1993). Cette diversification de la fréquentation alimente, par souci de distinction, une représentation très négative des «transports en commun », qui imprègne encore certaines approches normatives de la mobilité (Ferrarini, 1999 par exemple).

Ces visions de l'espace du train questionnent la combinaison de diversité, d'anonymat et de densité de passagers qui caractérise l'urbanité. Si l'espace du train est apparemment "surdéterminé de solitude collective » (Augé, 1992), il faut envisager des pratiques qui ne relèvent pas de relations sociales d'interconnaissance et valorisent cet environnement socio-technique contraignant.

\section{Un espace d'appropriation et de transgression}

Le train fait l'objet d'appropriations diverses, comme celles qui relèvent de son usage comme lieu de travail. Le train participe en effet à la division spatiale du travail concernant le personnel naviguant, mais aussi certains passagers. Giorgio par exemple apprécie d'y disposer de longues plages de travail, bien délimitées dans le temps et ne souffrant pas d'interruptions. A la manière du travail domestique, ce travail est peu visible socialement. Se pencher sur les formes de détournement de cet espace très fonctionnel permet de reconnaître une plus forte latitude d'action encore aux acteurs (Gillet, 2006). C'est le non-lieu mais au sens de Michel de Certeau cette fois : les "marcheurs innombrables ", ici les passagers, inventent des fonctionnalités nouvelles et subversives au cœur même du train, "lieu de l'urbaniste ». Symbolisé en Italie par la figure du vendeur ambulant : le bibitaro, le commerce informel participe de cette diversification fonctionnelle. Porté à l'écran par Nanni Loy dans Café Express en 1979, les bibitari sont toujours présents à bord des trains grandes lignes du sud de l'Italie. Dans les trains interrégionaux du nord-est, l'ambulantage est davantage le fait d'individus se présentant comme sourds-muets, déposant un petit gadget sur les sièges accompagné d'un mot incitant au don. Mais les pratiques déviantes ne sont 
pas seulement de nature commerciale. Certains voyageurs subvertissent l'interdiction de fumer, notamment dans les toilettes des Intercity, équivalent des trains Corail en Italie. D'autres alimentent le thème du voyage érotique : un magazine italien livre ainsi un véritable mode d'emploi spatial de l'amour dans le train sans être surpris du contrôleur (Lui Guidemagazine, 2006).

Ces transgressions placent le train en rupture des normes du territoire qu'il dessert. L'espace du train, tout en mettant en rapport différents lieux, suspend en quelque sorte, le temps du parcours, la liaison qu'il établit. Cette neutralisation de la liaison rappelle presque point par point la définition des hétéropies, emplacements «qui ont la curieuse propriété d'être en rapport avec tous les autres [...] mais sur un mode tel qu'ils suspendent, neutralisent ou inversent l'ensemble des rapports qui se trouvent, par eux, désignés, reflétés ou réfléchis " (Foucault, 1994, p. 755). Mais l'intérêt de l'hétérotopie réside moins dans l'assignation de notre objet à une catégorie que dans le questionnement qu'elle introduit sur le fonctionnement de l'espace du train. Elle incite à étudier son ouverture relative à l'espace traversé, ses différentes temporalités comme sa capacité à tenir ensemble divers lieux.

\section{Ni lieu, ni territoire : la territorialité de l'espace de transport}

Si le train n'est pas un non-lieu au sens de Marc Augé, on peut se demander s'il ne constitue pas à lui seul une sorte de lieu anthropologique ou de territoire, marqué par une limite matérielle et symbolique entre l'ici et l'ailleurs, entre soi et l'autre, associée à un certain nombre de représentations et de pratiques collectives. Le collectif des passagers pourrait bien être la «communauté juridique des voyageurs» (Terrolle, 1993) dont le principe d'appartenance repose sur l'acquisition d'un droit contractuel d'accès au train. Ainsi, le fraudeur qui refuse de régulariser sa situation se voit exclu du train à la gare suivante : le pouvoir du chef de train s'exerce, accepté de manière tacite par la communauté. Les relations d'usage du train sont aussi investies de significations particulières, réductibles à certaines aspirations communes: le train comme un espace public soumis à un droit d'accès, dans lequel une place est reconnue pour séjourner le temps d'accomplir un certain parcours.

Mais le critère d'une certaine durée de l'expérience territoriale, du temps long, manque à la communauté des voyageurs. Le temps de trajet est convenu d'avance et c'est précisément sa limitation qui rend socialement acceptable l'existence d'une telle communauté d'anonymes : elle se forme dans la coprésence, et non dans la coexistence. L'espace du train implique non pas un territoire mais une autre forme de territorialité, plus souple, plus plastique, une sorte de territoire provisoire où les places et leur appropriation sont remises en jeu après chaque trajet. C'est cette propriété qu'exploitent certains habitués des gares de Naples et Milan, ratissant les voitures à l'arrivée des trains pour y récupérer les effets personnels oubliés, même quelques instants (Colombo et al., 1999). Les traces de passage sont autant de signes pour les nouveaux passagers d'une présence plus ancienne. Le plus souvent évoquée pour ses aspects salissants, cette présence retient aussi la curiosité des passagers. Les inscriptions « divertissantes" ou les journaux "qu'on n'achèterait jamais » dont s'amusent Luca et Giorgia sont autant de signes tangibles d'une altérité persistante.

Pourrait-on alors considérer le train comme un lieu ? Cela semble difficile dans la mesure où le territoire contient les lieux qu'il unifie et que le train est partie prenante de la relation englobante. II participe peut-être à l'agencement symbolique du territoire. A la manière des mairies françaises ou des digues néerlandaises, ce serait alors un lieu générique représentant la cohésion sociale du territoire desservi, par une projection symbolique du territoire en ce lieu (Debarbieux, 1995). Mais le train assure plus nettement encore cette articulation de manière physique et fonctionnelle. On peut dès lors se demander s'il est possible de parler de lieu lorsque l'ancrage n'est pas fixe, ne relève pas de l'enracinement. Si oui, comment comprendre cette souplesse de l'ancrage en termes d'habiter? 


\section{2 - Habiter l'espace mobile}

«L'espace mobile » proposé par Denis Retaillé (2009, notamment) envisage précisément le lieu dégagé de l'enracinement, comme un mobile qui passe de site en site. Le train est dans cette perspective un lieu mobile passant à travers divers sites d'investissement matériel (gares). De même, à l'échelle du train lui-même, l'espace se compose de divers sites et ressources que s'approprient les passagers pour faire leur place dans le train. La place est l'ancrage caractéristique de cet espace mobile, sa construction pouvant absorber une part importante de l'attention des passagers. Le fonctionnement de la première classe semble reposer précisément sur une valorisation de la place, où l'« individualité [est] respectée et protégée dans et par l'espace », faisant apparaître une certaine reproduction de l'intérieur bourgeois (Canto-Klein, 1975). L'espace domestique est alors érigé en modèle de la place. Les observations et les entretiens réalisés en seconde classe dans le cas italien révèlent en revanche des constructions moins sédentaires.

\section{Prendre place}

Investissement de composantes fixes sur lesquelles le corps s'appuie, la place par excellence s'articule autour de l'assise prévue par le transporteur. L'individualisation de la place assise est cependant une construction historique récente pour les trains des navetteurs. C'est seulement en 1997 que, pour la première fois en Italie, le designer Sergio Pininfarina remplace les banquettes par des sièges individuels à bord des Trains à Forte Fréquentation.

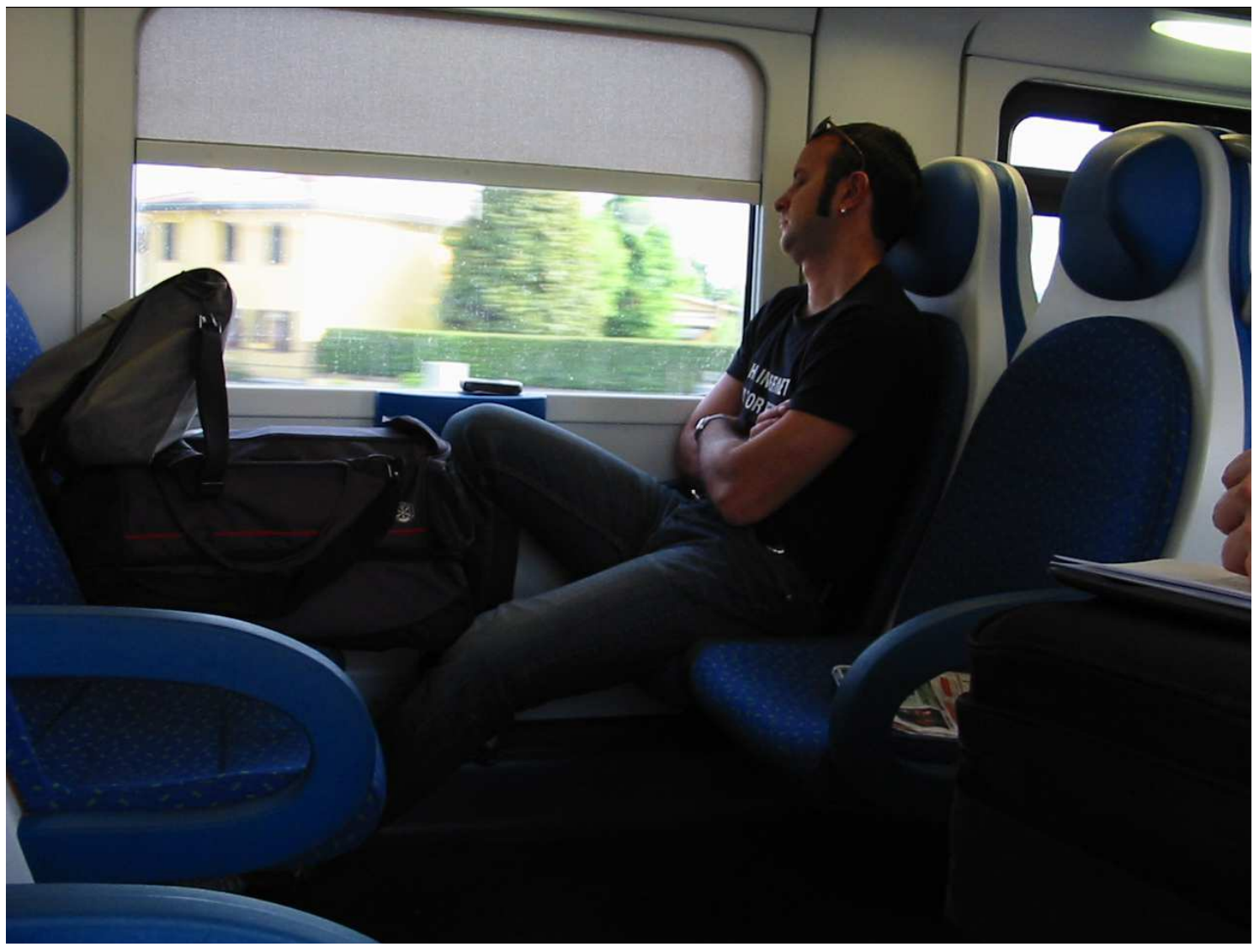

Document 1 - L'extension d'une place, ancrage éphémère à bord du Train à Haute Fréquentation entre Padoue et Venise (avril 2006). Photographie de l'auteur. 
C'est dans un de ces trains qu'a été pris, en période creuse, le cliché du document 1. S'y distingue l'organisation de l'espace personnel du dormeur. La tête est appuyée sur l'oreillette unique, et non posée, car celle-ci est trop étroite. Le train a été conçu pour des trajets courts où le passage l'emporte sur le confort, d'où un certain raidissement du port de tête, incorporant cette contrainte socio-technique. Une jambe est posée sur le rebord de la plinthe. Les bagages sont placés en vis-à-vis. A portée de main, le journal est placé sur le siège voisin et le portable sur la petite console de la fenêtre, dans le prolongement du regard.

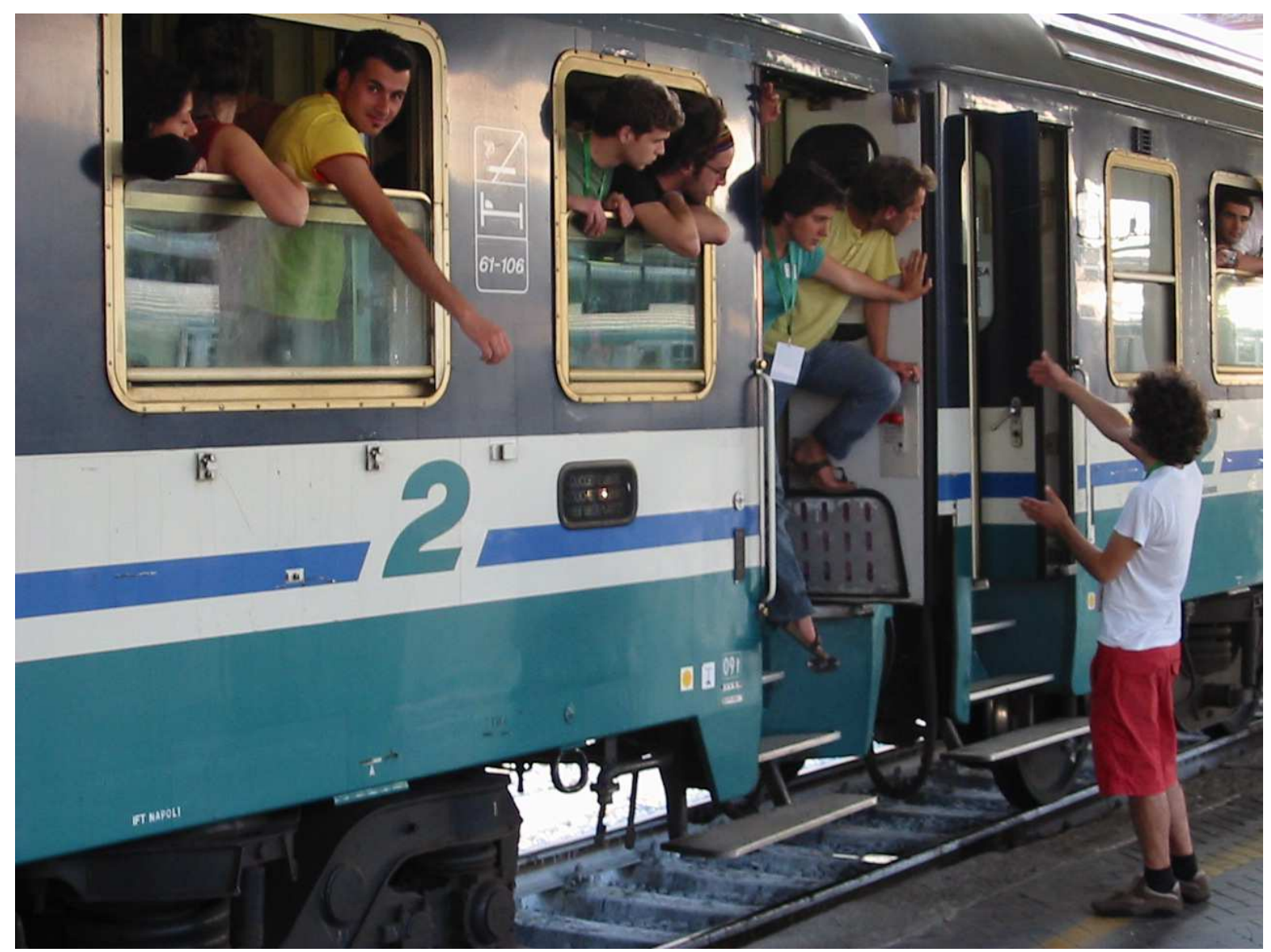

Document 2 - La création de places surnuméraires à bord d'un train grande ligne: exemple en gare de Bologne (mai 2006).

Photographie de l'auteur.

Des places autres qu'assises servent aussi de point d'ancrage. Matériellement, elles limitent les effets d'oscillation de la voiture pour le corps. Symboliquement, elles assurent la position. Poignée, barre d'appui, mur contre lequel on s'adosse, marche d'escalier ou valise sont investis. A bord d'un Intercity, l'essentiel des places surnuméraires se crée dans le couloir des voitures à compartiment. Lorsque les strapontins prévus à cet effet sont occupés, les fenêtres constituent un support privilégié de place, comme l'atteste la photographie du document 2.

La fenêtre fonctionne plus généralement comme une échappatoire spatiale et temporelle à l'espace intérieur. Elle conjure l'étroite limite de la place accordée au passager en réinscrivant le voyage dans un référentiel extérieur au train. Elle évite la confrontation visuelle directe entre passagers, comme Fellini l'a montré dans son film publicitaire $O h$, che bel paesaggio en 1984. Ennuyée par la conversation d'un homme qui lui fait face, une jeune fille blonde s'empare d'une télécommande et change la scène qu'on entrevoit de la fenêtre : 
les panoramas les plus célèbres d'Italie défilent, jusqu'à la place des Miracles de Pise, où se découpe la bouteille d'un célèbre apéritif italien.

La communication de la grande entreprise d'exploitation ferroviaire italienne, Trenitalia, témoigne en creux de l'aspiration à une large place personnelle. Elle privilégie une vision de l'espace du train presque vide, le plus souvent réduit à la présence d'un ou deux passagers. Une de ses publicités (document 3) manifeste à l'extrême la négation du rapport à l'autre et à la place qui lui est accordée. La question au centre de l'affiche, "Comment est le paysage à 300 à l'heure ? ", peut difficilement être attribuée à un interlocuteur à bord du train. Elle renvoie à une expérience physique et esthétique individuelle, et à sa possible évocation ultérieure, lors d'une discussion hors de l'espace du train, dans un registre de langage familier.

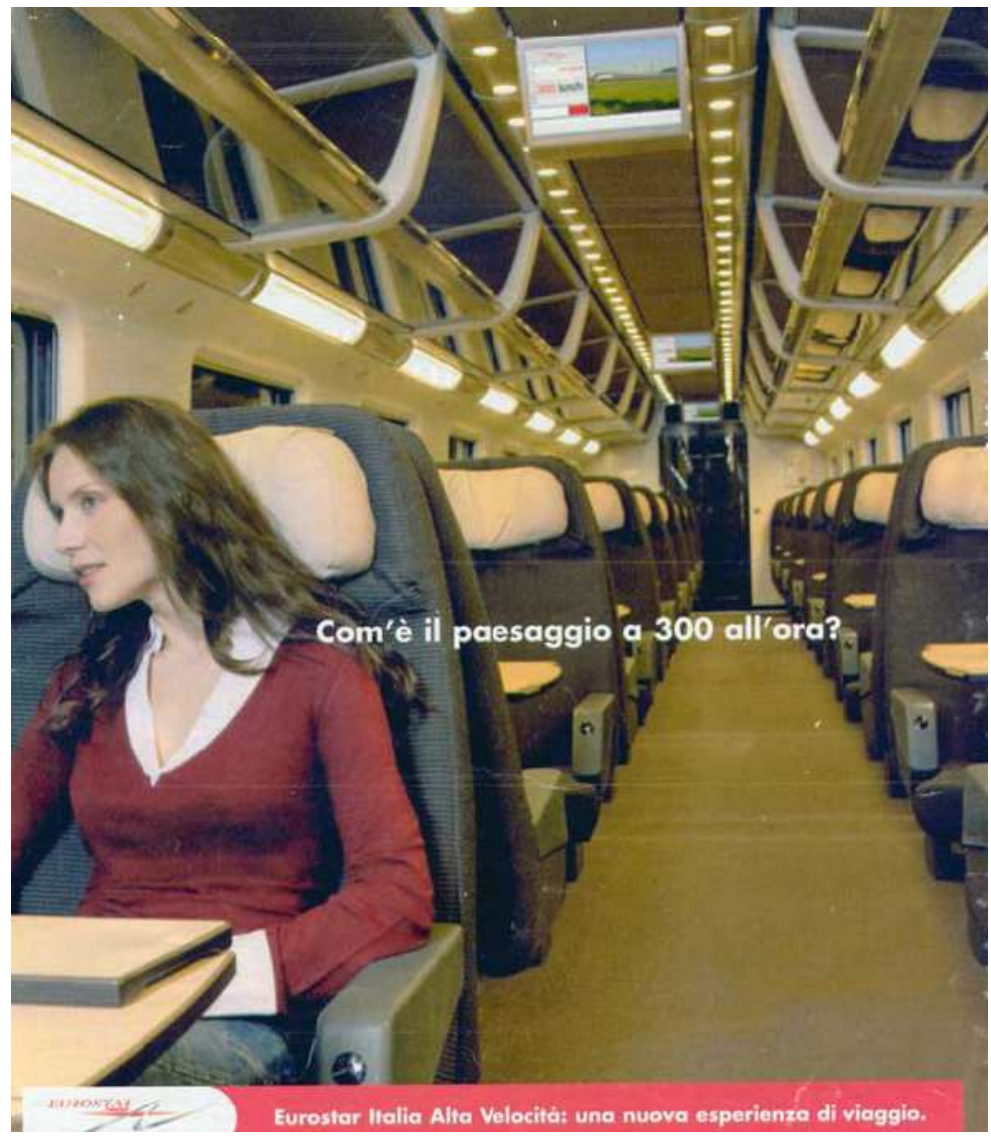

Document 3 - «Comment est le paysage à 300 à l'heure ? », publicité pour Trenitalia dans le supplément « Venerdi » de la Repubblica (mars 2006).

\section{Pouvoir et mise en place}

L'attribution des places dans le train est au cœur de diverses stratégies des passagers, dans une sorte de lutte des places, pour reprendre, et réactiver dans son sens le plus littéral, une expression employée par Michel Lussault (2009). En témoigne le règlement des chemins de fer italien, qui encadre très attentivement l'usage de la place assise. Le droit d'occuper la place réservée n'est par exemple assuré "que jusqu'à 15 minutes après le départ du train de la gare à laquelle la réservation prend effet » (R.D.L., art.3). Mais les effets personnels sont autant de marqueurs d'appropriation reconnus : «le voyageur qui, au départ ou durant le voyage, abandonne provisoirement sa place, a le droit de la réoccuper quand y est laissé un objet personnel » (R.D.L., art.4, §3). La simulation d'occupation de place est d'ailleurs théoriquement sanctionnée, ce qui révèle en creux l'existence et l'efficacité de la 
pratique. L'écrivain sicilien Leonardo Sciascia l'évoque dans une de ses nouvelles : « il suffit d'éteindre la lumière, de baisser les rideaux et de répartir bagages et journaux sur les banquettes » (1977, p. 31). Mais « contaminer » un siège en y posant ses pieds, prononcer quelques mots ou détourner le regard suffit souvent à décourager le passager en quête de siège.

Le choix d'une place renvoie à une hiérarchie implicite des positionnements, accordant à chaque place une valeur différentielle. Ivana par exemple "choisi[t] l'étage supérieur pour mieux voir le paysage ». Luca et Giulia le privilégient pour éprouver « un plaisir d'enfant » que le choix de nombreux groupes scolaires corrobore. Francesca en apprécie la relative tranquillité, l'étage surbaissé étant souvent plus fréquenté. Mais c'est l'étage inférieur que préfère Cecilia, parce qu'il ménage plus d'espace, se trouve plus proche de la sortie et limite le transport des bagages. Autre manifestation de cette mise en place, dans le train de Fabio, navetteur depuis six ans en Vénétie: "les habitués vont chacun à leur place après s'être salués ». Les gestes de ces passagers, parfaitement calculés, révèlent l'ampleur de la connaissance pratique de l'espace du train qu'ils ont progressivement incorporée.

La hiérarchie des places n'est pas seulement individuelle, mais aussi collective. Dans le train de retour de Fabio, en été, la lutte des places se concentre sur les sièges à l'ombre jouxtant les fenêtres. La compétition pour les places est souvent discrète, mais d'une mesure parfois très précise : "à chaque fois que mon voisin tournait les pages de son journal, raconte Fabio, il repoussait un peu plus mon bras de l'accoudoir ». La communauté des voyageurs observe par ailleurs une certaine protection de la place du voyageur qui s'absente. Des antagonismes plus manifestes se substituent toutefois parfois à ces rapports feutrés de régulation. La présence de groupes scolaires ou sportifs concurrence le fonctionnement de la communauté, tout comme une forte affluence. Lorsqu'un train est annulé, ses passagers contestent parfois ouvertement la propriété des places du train sur lequel ils se rabattent pour s'en emparer. Les trains-couchettes italiens, dont les compartiments ne sont pas mixtes, connaissent la même situation. Lorsque la place vient à manquer dans les compartiments masculins, les passagers s'emparent de fait des couchettes vacantes des compartiments féminins, la situation réciproque se présentant très rarement. Une telle asymétrie manifeste une relation genrée de pouvoir qui réactive, mais aussi recontextualise, des normes caractéristiques des territoires desservis par le train. Elle invite à replacer les interactions qui s'y opèrent à plus large échelle.

\section{3 - Une cohabitation à large échelle dans un micro-espace}

Ponctionnant à chaque gare une part de la population présente localement, le train opère une sorte de transect du territoire traversé. Ce dispositif est particulièrement prégnant dans le cas d'un réseau urbain multipolaire comme celui de l'Italie, où les arrêts intermédiaires sont nombreux. Le train rassemble ainsi provisoirement à micro-échelle des trajectoires de personnages mobiles qui divergent à plus large échelle. II est donc nécessaire d'examiner le rôle de ce jeu d'échelle dans la cohabitation provisoire des passagers.

\section{Sociabilité et désattention}

L'ambivalence de la territorialité du train laisse aux voyageurs un large choix de pas de mesure pour y définir les distances interpersonnelles. Jouant de la proximité physique, ils minimisent ces distances ou, au contraire, les augmentent, marquant le caractère incident de leur coprésence. Plus ou moins conscient, cet arbitrage est au cœur du dispositif d'urbanité et se révèle souvent socialement discriminant.

Les passagers adoptent dans certaines situations des mesures de la distance favorables à la sociabilité, même entre inconnus. L'égalité face à la technique du transport collectif sert par exemple de ciment face à l'annonce d'un retard. La formule selon laquelle, 
« ce ne sont pas seulement les distances entre les lieux, mais les écarts entre les hommes qui diminuent» (Pecqueur, 1839) recouvre alors une certaine réalité. Elle n'est pas sans rappeler le rôle politique dévolu au réseau ferroviaire dans l'unification italienne. Le cinéaste Nanni Loy a saisi toute la richesse d'un tel dispositif. II s'en empare en 1977 dans Voyage en seconde classe, un de ses programmes pour la télévision. En caméra cachée dans un compartiment d'un train sillonnant l'Italie, il cherche à "mettre ensemble des fragments d'une Italie à certains égards inconnue ». Se faisant passer pour des voyageurs ordinaires, ses collaborateurs et lui-même lancent des sujets qui visent à susciter, souvent avec succès, des débats enflammés entre les voyageurs d'horizons très différents de la péninsule, rassemblés dans le compartiment.

Les voyageurs marquent bien souvent en revanche une certaine distance sociale, voire une soigneuse désattention civile envers certaines minorités, notamment à l'égard des vendeurs ambulants sénégalais, très présents dans les villes italiennes. Souvent appelés les "Marocains" ou de manière condescendante les "vuccompra», on les retrouve fréquemment isolés dans un carré ou dans un salon des trains régionaux toscans. Rass, navetteur sénégalais, se voit ainsi signifier clairement par certains passagers qu'il devrait se tenir à distance. "Lorsqu'il n'y a plus de place ailleurs, je n'ai pas le choix » se justifie-t-il, intégrant ainsi dans son choix de placement la contrainte de séparation souhaitée par son voisinage.

Mais la sociabilité se cristallise aussi dans la pratique de l'entretien. Comme les autres passagers, le chercheur y fait figure d'étranger (Matthey, 2005), susceptible d'émettre seulement des jugements sociaux décalés qui n'auront pas vraiment d'écho dans le monde social de celui qui s'y prête. II constitue alors une figure d'objectivité pour l'interlocuteur, qui peut mettre à distance son rôle social. L'effet de rupture du train par rapport au territoire desservi favorise non seulement le bon déroulement de l'entretien mais se révèle aussi à travers lui.

\section{Deux modèles spatiaux de confrontation à l'altérité}

Le rôle du train dans la cohabitation provisoire des passagers peut plus précisément s'analyser en termes de cadre d'interaction. Le système de références spatiales mobilisé par les acteurs s'insère dans une étiquette de la rencontre et de la conversation que les interactionnistes cherchent in fine à décrire (Goffman, 1996). Mais c'est ici ce système luimême qui retient l'attention. Cette "scène " offre différentes configurations spatiales au déploiement de la sociabilité.

Le compartiment et le salon forment deux configurations intérieures du train dont les grandes lignes n'ont pas connu de réelle évolution dans l'histoire ferroviaire. Elles condensent deux conceptions très différentes des relations sociales dans l'espace de transport. L'agencement du compartiment est calqué sur le modèle en $U$ de la diligence, luimême issu de la juxtaposition de deux chaises à porteur. Le compartiment s'inscrit dans une tradition européenne de la "conversation de voyage » en face-à-face. Au contraire, le modèle du salon, créé aux États-Unis, s'organise autour d'un couloir central dans l'espace ouvert de la voiture. Les sièges sont orientés dans une même direction, selon le modèle Pullman adopté pour les autocars.

Dans son Histoire du voyage en chemin de fer, Wolfgang Schivelbusch (1990) affiche sa préférence pour le modèle du salon, au nom de considérations techniques (chauffage et installations sanitaires) mais aussi de sécurité, source considérable de préoccupations dès les premiers temps du chemin de fer. En revanche «pour le public européen, dit-il, c'est le compartiment qui est et qui demeure le véritable espace de voyage, c'est pour en conserver la paix et l'isolement, également sources de plaisir et d'angoisse, que le $19^{\mathrm{è}}$ siècle a produit une disposition de l'espace aussi monstrueuse [...] et combien peu "naturelle" » (1990, p. 93).

C'est en dernière instance un jugement social sur les deux dispositifs qui l'emporte dans le choix de l'historien. Chacun d'eux renvoie au fond à un modèle de confrontation à 
l'altérité. Le premier passe par un face-à-face avec autrui, caractéristique du voyage. Le second opère dans le côtoiement, relevant davantage des codes urbains feutrés de l'inattention civile. Trop explicitement consacré à la conversation, le compartiment créerait pour Schivelbusch une sorte de malaise du huis-clos entre inconnus, alors que le décloisonnement de l'espace du salon, offrant une médiation à l'altérité, s'accompagnerait d'une certaine ouverture de chacun aux autres. Peut-on pour autant parler d'un modèle sociofuge, défavorable à la sociabilité, caractéristique du compartiment, opposé à un modèle sociopète du salon?

Le discours des passagers italiens tendrait en première instance à inverser l'analyse. Le salon des trains à grande vitesse italiens (Eurostar) est décrit dans les entretiens comme un espace défavorable à la sociabilité, par opposition au compartiment de l'Intercity. La réversibilité du caractère favorable d'une forme au développement de la sociabilité incite à douter de la pertinence de ces qualifications indépendamment du sens et des normes que

les acteurs accordent à ces dispositifs. Dans le cas italien, ces jugements entre compartiment et salon renvoient aussi aux différences entre Eurostar et Intercity. Diverses caractéristiques pourraient jouer en fait en faveur d'une sociabilité accrue de I'Intercity, comme son moindre coût d'accès, la plus longue durée des trajets proposés, la fréquence des arrêts ou le confort des fauteuils. Ces contrastes renvoient au travail, particulièrement intense, de stratification des catégories et donc des espaces du train. Une telle perspective rappelle la nécessaire prise en compte des logiques à plus large échelle qui l'informent.

\section{Conclusion : L'espace du train, laboratoire d'urbanité}

Espace de mobilité, l'espace du train n'est pas, à l'analyse, un espace neutre. Les discours qui en nient l'intérêt tendent au contraire à souligner la pertinence de son étude. Par la déliaison provisoire mais répétée que la mobilité implique, le train renvoie à une territorialité plus souple que celle d'un lieu ou d'un territoire stricto sensu. C'est ce décalage qui confère au train une marge d'incertitude sur son fonctionnement.

C'est dans la coprésence et l'anonymat que l'habiter mobile se construit, autour de la délimitation de la place de chacun, plus ou moins régulée par la communauté des voyageurs. Coprésence et anonymat permettent la formation souple, mais socialement contrastée, d'interactions de cohabitation entre passagers. Les relations sont établies ou non avec autrui de manière sélective, selon la mesure de la distance adoptée. C'est ce qui en fait un espace d'urbanité (Lévy, Lussault, 2003, art. Coprésence).

Le train constitue en quelque sorte un laboratoire d'urbanité : la relation sociale de soi aux autres dans l'espace s'y réinvente, dans l'expérience quotidienne des navetteurs comme dans celle des passagers occasionnels. C'est à ce titre que l'espace du train constitue un objet légitime de connaissance : s'y retravaillent les fondements du fonctionnement du lieu et du territoire par la mobilité et l'habiter.

\section{Note :}

(1) Les noms des interlocuteurs employés ici sont fictifs pour garantir leur anonymat. Travail effectué dans le cadre d'un mémoire de Master 1: L'espace du train en Italie dans une perspective de géographie culturelle (2006), sous la direction de Béatrice Collignon, Université Paris I. Disponible à la Bibliothèque de l'Institut de Géographie de Paris et sur : http://www.geographie.ens.fr/IMG/file/Memoires eleves/Fretigny\%20M1.pdf

Je remercie chaleureusement Béatrice Collignon pour son aide précieuse dans la réalisation de ce travail ainsi que Nadine Cattan pour ses fructueuses remarques sur le texte. 


\section{Bibliographie :}

Augé M. (1992), Non-lieux, Paris, Seuil.

Berry M. et Hamilton M. (2010), “ Changing Urban Spaces: Mobile Phones on Trains », Mobilities, n 5-1, p. 111-129.

Binnie J. et al. (2007), « Mundane mobilities, banal travels », Social \& Cultural Geography, n 8-2, p. 165-174.

Bissell D. (2010), «Passenger mobilities: affective atmospheres and the sociality of public transport », Environment and Planning D: Society and Space, n 28-2, p. 270-289.

Bissell D. (2009), « Visualising everyday geographies: practices of vision through traveltime », Transactions of the Institute of British Geographers, n 34-1, p. 42-60.

Canto-Klein M. (1975), «A propos de la représentation de l'espace et du temps », Cahiers internationaux de la sociologie, juillet 1975, n 59-D, Évry, PUF, p.355-366.

Certeau de M. (1990), Arts de faire, Paris, Gallimard.

Collignon B. et Staszak J.-F. (dir.) (2003), Espaces domestiques : construire, habiter et représenter, Paris, Bréal.

Colombo E. et Navarini G. (1999), Confini dentro la città, Milan, Angelo Guerini e Associati. Debarbieux B. (1995), «Le lieu, le territoire et trois figures de rhétorique», L'Espace géographique, 2, p. 97-112.

Ferrarini A. (1999), Forme e icone del moderno, Bologne, Pendragon.

Foucault M. (1994), « Des espaces autres », in Dits et écrits 4, Paris, Gallimard, p. 752-762.

Gillet A. (2006), « Dérives atopiques », EspacesTemps.net, 08 mai 2006. www.espacestemps.net/document1975.html

Goffman E. (1996), Les relations en public, Paris, Éditions de Minuit.

Lannéelle X. (2004), « Va et vient. Entre deux lieux, entre recherche et récit », EspacesTemps.net, Textuel, 7 avril 2004. http://espacestemps.net/document153.html

Lévy J. et Lussault M. (dir.) (2003), Dictionnaire de la géographie, Paris, Belin. Lussault M. (2009), De la lutte des classes à la lutte des places, Paris, B. Grasset. Lui Guidemagazine (2006), « Sesso al letto ? No, grazie », mai 2006, n 5.

Lyons G. (2007), « The use of travel time by rail passengers in Great Britain », Transportation Research, n 41-A, p. 107-120.

Matthey L. (2005), «Éthique, politique et esthétique du terrain : cinq figures de l'entretien compréhensif », Cybergeo, n 312, 31 mai 2005, 19 p.

http://cybergeo.revues.org/index3426.html

Pecqueur C. (1839), Économie sociale, Paris, Desessart.

Retaillé, D. (2009), « Malaise dans la géographie : l'espace est mobile », in Vanier M. (dir.), Territoires, territorialité, territorialisation, Rennes, P.U.R., coll. Espace et territoires.

R.D.L. (Regio decrete legge), (1934), Condizioni di trasporti, section : Normativa per il servizio interno, 11 octobre 1934, n 1948.

Sciascia L. (1977), “ La mer couleur de vin », in La mer couleur de vin, Paris, Denoël, p. 3169.

Schivelbusch W., (1990), Histoire des voyages en train, Paris, Le Promeneur.

Sheller M. et Urry J. (2006), « The new mobilities paradigm », Environment and Planning A, n 38-2, p. 207-226.

Stock M. (2005), “ Les sociétés à individus mobiles : vers un nouveau mode d'habiter ? », EspacesTemps.net, 25 mai 2005. www.espacestemps.net/document1353.html

Terrolle D. (1993), “'Entre-deux' », in Petonnet C. et Delaporte Y. (dir.), Ferveurs contemporaines. Paris, L'Harmattan, p.243-259.

Urbain J.-D. (1993), L'ldiot du voyage : histoires de touristes, Paris, Payot.

Watts L. (2008), « The Art and Craft of Train Travel », Social \& Cultural Geography, n 9 -6, p. 711-726. 